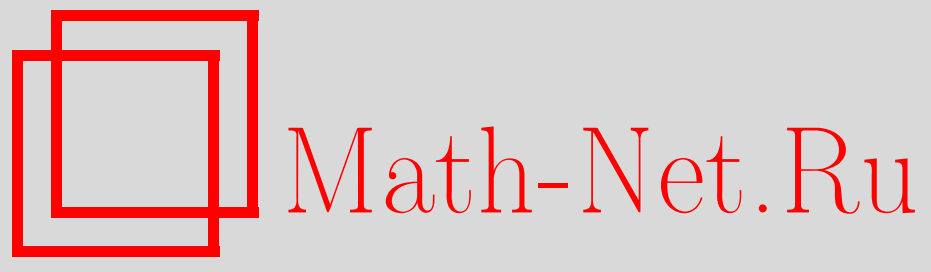

Е. Д. Лившиц, О жадном алгоритме для словарей с ограниченной совокупной когерентностью, Матем. заметки, 2010, том 87, выпуск 5, 792-795

DOI: https://doi.org/10.4213/mzm8722

Использование Общероссийского математического портала Math-Net.Ru подразумевает, что вы прочитали и согласны с пользовательским соглашением http://www . mathnet.ru/rus/agreement

Параметры загрузки:

IP : 3.85 .183 .62

26 апреля 2023 г., 11:06:49

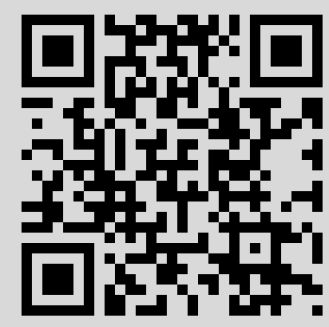




\section{О жадном алгоритме для словарей с ограниченной совокупной когерентностью}

\section{Е. Д. Лившиц}

Пусть $H=(H,\langle\cdot, \cdot\rangle)$ - действительное сепарабельное гильбертово пространство. Множество $\mathscr{D} \subset H$ называют словарем, если для любого $g \in \mathscr{D}$ выполнено $\|g\|=1$ и $\overline{\operatorname{span}} \mathscr{D}=H$. В последние годы большую значимость получила задача построения по элементу $f \in H$ конечной линейной комбинации элементов словаря $\sum_{k=1}^{m} c_{k}(f) g_{k}(f)$, $c_{k}(f) \in \mathbb{R}, g_{k}(f) \in \mathscr{D}$, достаточно хорошо приближающей $f$. Эта задача достаточно эффективно решается с помощью жадных алгоритмов [1]. Ниже приведены две наиболее известные их модификации.

ЧИСТО ЖАДНЫЙ АЛГОРИТм (ЧЖА). Пусть задана челевая функиия

$$
f_{0}^{\mathrm{PGA}}:=f_{0}:=f \in H \text {. }
$$

Для каждого $m \geqslant 0$ последовательно выбираем вектор $g_{m+1} \in \mathscr{D}$ такой, что

$$
\left|\left\langle f_{m}, g_{m+1}\right\rangle\right|=\sup _{g \in \mathscr{D}}\left|\left\langle f_{m}, g\right\rangle\right|
$$

и определяем

$$
f_{m+1}:=f_{m+1}^{\mathrm{PGA}}:=f_{m}-\left\langle f_{m}, g_{m+1}\right\rangle g_{m+1} .
$$

ОРТОГОНАЛЬНЫЙ ЖАДНЫЙ АЛГОРИТм (ОЖА). Пусть задана челевая функиия

$$
f_{0}^{\mathrm{OGA}}:=f \in H \text {. }
$$

Для каждого $m \geqslant 0$ последователъно выбираем вектор $g_{m+1}^{\mathrm{OGA}} \in \mathscr{D}$ такой, что

$$
\left|\left\langle f_{m}^{\mathrm{OGA}}, g_{m+1}^{\mathrm{OGA}}\right\rangle\right|=\sup _{g \in \mathscr{D}}\left|\left\langle f_{m}^{\mathrm{OGA}}, g\right\rangle\right|,
$$

и определяем

$$
G_{m+1}^{\mathrm{OGA}}=\operatorname{Proj}_{g_{1}^{\mathrm{OGA}}}, \ldots, g_{m+1}^{\mathrm{OGA}}(f), \quad f_{m+1}^{\mathrm{OGA}}:=f-G_{m+1}^{\mathrm{OGA}} .
$$

Тем самым, для каждого $m \geqslant 1$ построены $m$-членные приближения к $f$ :

$$
\sum_{k=1}^{m}\left\langle f_{k-1}, g_{k}\right\rangle g_{k} \quad \text { и } \quad G_{m}^{\mathrm{OGA}} .
$$

В настоящей статье будет изучаться скорость сходимости жадных алгоритмов для функций из класса $\mathscr{A}_{0}(\mathscr{D})$ - конечных линейных комбинаций элемента словаря и классов $\mathscr{A}^{p}(\mathscr{D}), 1 \leqslant p<2$. Положим для $M \geqslant 0$

$$
\mathscr{A}^{p}(\mathscr{D}, M):=\overline{\left\{\sum_{\lambda \in \Lambda} c_{\lambda} g^{\lambda}: \sum_{\lambda \in \Lambda}\left|c_{\lambda}\right|^{p} \leqslant M^{p}, c_{\lambda} \in \mathbb{R}, g^{\lambda} \in \mathscr{D}, \sharp \Lambda<\infty\right\}},
$$

где замыкание берется в норме пространства $H$. Пусть

$$
\begin{gathered}
\mathscr{A}^{p}(\mathscr{D}):=\bigcup_{M \geqslant 0} \mathscr{A}^{p}(\mathscr{D}, M), \\
|f|_{p}:=|f|_{\mathscr{A}^{p}(\mathscr{D})}:=\inf \left\{M \geqslant 0: f \in \mathscr{A}^{p}(\mathscr{D}, M)\right\}, \quad f \in \mathscr{A}^{p}(\mathscr{D}) .
\end{gathered}
$$

Работа выполнена при поддержке Российского фонда фундаментальных исследований (гранты №№ 08-01-00799 и 09-01-12173).

(C) Е. Д. Лившиц, 2010 
Из результатов ДеВора, Темлякова и автора [2], [3] вытекает, что в классе $\mathscr{A}^{1}(\mathscr{D})$ ОЖА дает оптимальную (для этого класса) скорость сходимости $C|f|{ }_{1} m^{-1 / 2}$, а ЧЖА нет. На более узких классах, например $A_{0}(\mathscr{D})$, скорость сходимости ОЖА не превышает $C m^{-1 / 2}$ и не является оптимальной. В то же время, если словарь $\mathscr{D}$ обладает некоторыми дополнительными свойствами, жадные алгоритмы могут сходиться значительно быстрее. Это направление, называемое разреженными аппроксимациями (sparse approximation), в последнее время интенсивно развивается (см. [4]-[7] и др.). Следуя Троппу [6], будем называть совокупной когерентностью словаря $\mathscr{D}$ величину

$$
\mu_{1}(\mathscr{D}):=\sup _{g \in \mathscr{D}} \sum_{\tilde{g} \in \mathscr{D}, \tilde{g} \neq g}|\langle\widetilde{g}, g\rangle|
$$

В вышеуказанных работах доказывался следующий базовый результат.

Tеорема 1. Пустъ заданы словаръ $\mathscr{D}$ с $\mu_{1}(\mathscr{D})<1 / 2 u f \in \mathscr{A}_{0}(\mathscr{D})$. Тогда

$$
G_{m}^{\mathrm{OGA}}(f, \mathscr{D})=f, \quad m \geqslant m_{0}, \quad\left\|f_{m}\right\| \leqslant C \exp (-c(f) m), \quad m \geqslant 0, \quad c(f)>0 .
$$

Для словарей с малой совокупной когерентностью ЧЖА оптимален в $\mathscr{A}^{p}(\mathscr{D})$, если же $\mu_{1}(\mathscr{D})$ велико, то оптимальная скорость может не достигаться.

Теорема 2. Пустъ заданы словаръ $\mathscr{D}$ с $\mu_{1}(\mathscr{D})<1 / 3, p, 1 \leqslant p<2 u f \in \mathscr{A}^{p}(\mathscr{D})$. Тогда

$$
\left\|f_{m}^{\mathrm{PGA}}\right\| \leqslant C\left(p, \mu_{1}(\mathscr{D})\right)|f|_{p} m^{-1 / p+1 / 2} \quad \partial \Omega \Re \quad m \geqslant 1 .
$$

Tеорема 3. Пустъ заданы словаръ $\mathscr{D}$ с $\mu_{1}(\mathscr{D})<1 / 3 u f \in \mathscr{A}^{1}(\mathscr{D})$. Тогда

$$
\left\|f_{m}^{\mathrm{PGA}}\right\| \leqslant|f|_{1} m^{-1 / 2} \quad \text { для } m \geqslant 1 .
$$

Tеорема 4. Существует словаръ $\mathscr{D}$ с $\mu_{1}(\mathscr{D})<\infty, f_{0} \in \mathscr{A}_{0}(\mathscr{D}), \beta>0$ u $C>0$ mакой, что для любого $m \geqslant 1$

$$
\left\|f_{m}^{\mathrm{PGA}}\right\| \geqslant C m^{-1 / 2+\beta} .
$$

Приведем основные шаги доказательства теорем 2 и 3. Если не оговорено противное, будем считать, что $c_{\lambda} \in \mathbb{R}, g^{\lambda} \in \mathscr{D}, \lambda \in \Lambda$. Следующие две леммы являются стандартными в теории разреженных аппроксимаций.

Лемма 1. Пустъ заданы словарь $\mathscr{D}$ с $\mu_{1}(\mathscr{D})<1 / 2$ и конечное $\Lambda \subset \mathbb{N}$. Тогда имеет место неравенство

$$
\left(1-2 \mu_{1}(\mathscr{D})\right) \sum_{\lambda \in \Lambda} c_{\lambda}^{2} \leqslant\left\|\sum_{\lambda \in \Lambda} c_{\lambda} g^{\lambda}\right\|^{2} \leqslant\left(1+2 \mu_{1}(\mathscr{D})\right) \sum_{\lambda \in \Lambda} c_{\lambda}^{2} .
$$

Лемма 2. Пусть задано конечное множество индексов $\Lambda \subset \mathbb{N} u \epsilon>0$. Если для элемента $f$ имеет место представление

$$
f=f_{\epsilon}+\sum_{\lambda \in \Lambda} c_{\lambda} g^{\lambda}, \quad \sum_{\lambda \in \Lambda}\left|c_{\lambda}\right|^{p}=|f|_{p}^{p}, \quad\left\|f_{\epsilon}\right\|<\epsilon
$$

то справедливы неравенства

$$
\begin{array}{rll}
\left|\left\langle f, g^{\lambda_{0}}\right\rangle-c_{\lambda_{0}}\right|<\mu_{1}(\mathscr{D}) \max _{\lambda \in \Lambda}\left|c_{\lambda}\right|+\epsilon & \partial \Omega \Omega & \lambda_{0} \in \Lambda, \\
\left|\left\langle f, g^{\lambda_{0}}\right\rangle\right|<\mu_{1}(\mathscr{D}) \max _{\lambda \in \Lambda}\left|c_{\lambda}\right|+\epsilon & \partial \Omega \Omega & \lambda_{0} \notin \Lambda .
\end{array}
$$


Лемма 3. Пусть задан словарь $\mathscr{D}$ с $\mu_{1}(\mathscr{D})<1 / 3, f \in \mathscr{A}^{p}(\mathscr{D})$ u $m \geqslant 1$. Предположим, что для $n=m-1$, конечного $\Lambda \subset \mathbb{N} u \epsilon>0$ имеет место представление

$$
f_{n}=f_{n}^{\mathrm{PGA}}(f, \mathscr{D})=f_{\epsilon}+\sum_{\lambda \in \Lambda} c_{\lambda, n} g^{\lambda}, \quad c_{\lambda, n} \in \mathbb{R}, \quad g^{\lambda} \in \mathscr{D}, \quad\left\|f_{\epsilon}\right\|<\epsilon .
$$

Если $\epsilon<(1 / 6)\left(1-3 \mu_{1}(\mathscr{D})\right) \max _{\lambda \in \Lambda}\left|c_{\lambda, m-1}\right|$, то для $n=m$ будет иметь место представление (3) с теми же $\Lambda$ и $f_{\epsilon}$ и будут выполнятвся неравенства

$$
\begin{gathered}
\sum_{\lambda \in \Lambda}\left|c_{\lambda, m}\right|^{p} \leqslant \sum_{\lambda \in \Lambda}\left|c_{\lambda, m-1}\right|^{p}-2^{-p}\left(1-3 \mu_{1}(\mathscr{D})\right)^{p} \max _{\lambda \in \Lambda}\left|c_{\lambda, m-1}\right|^{p}, \\
\max _{\lambda \in \Lambda}\left|c_{\lambda, m}\right| \leqslant \max _{\lambda \in \Lambda}\left|c_{\lambda, m-1}\right| .
\end{gathered}
$$

ДокАЗАТЕЛЬСтво ЛЕммы 3. С помощью леммы 2 устанавливается, что существует $\lambda_{0} \in \Lambda$ для которого

$$
\begin{gathered}
\left|\left\langle f, g^{\lambda_{0}}\right\rangle\right|=\sup _{g \in \mathscr{D}}|\langle f, g\rangle|>\left(1-\mu_{1}(\mathscr{D})\right) \max _{\lambda \in \Lambda}\left|c_{\lambda, m-1}\right|-\epsilon, \\
\left|c_{\lambda_{0}, m-1}\right|>\left(1-2 \mu_{1}(\mathscr{D})\right) \max _{\lambda \in \Lambda}\left|c_{\lambda, m-1}\right|-2 \epsilon .
\end{gathered}
$$

Отсюда по лемме 2 выводим, что для $f_{m}$ имеет место представление (3) с

$$
c_{\lambda, m}=c_{\lambda, m-1}, \quad \lambda \in \Lambda \backslash\left\{\lambda_{0}\right\}, \quad c_{\lambda_{0}, m}=c_{\lambda_{0}, m-1}-\left\langle f, g^{\lambda_{0}}\right\rangle .
$$

При этом

$$
\begin{aligned}
\left|c_{\lambda_{0}, m}\right| & \leqslant\left|c_{\lambda_{0}, m-1}\right|-\left(1-3 \mu_{1}(\mathscr{D})\right) \max _{\lambda \in \Lambda}\left|c_{\lambda, m-1}\right|+3 \epsilon \\
& \leqslant\left|c_{\lambda_{0}, m-1}\right|-2^{-1}\left(1-3 \mu_{1}(\mathscr{D})\right) \max _{\lambda \in \Lambda}\left|c_{\lambda, m-1}\right|, \\
\left|c_{\lambda_{0}, m}\right|^{p} & \leqslant\left|c_{\lambda_{0}, m-1}\right|^{p}-2^{-p}\left(1-3 \mu_{1}(\mathscr{D})\right)^{p} \max _{\lambda \in \Lambda}\left|c_{\lambda, m-1}\right|^{p} .
\end{aligned}
$$

ДоКАЗАТЕЛЬСТво теОРемы 2 . Пусть $k \geqslant 1$. Для произвольного $\epsilon$

$$
0<\epsilon<\frac{1}{6}\left(1-3 \mu_{1}(\mathscr{D})\right) k^{-1 / p}|f|_{p},
$$

для элемента $f$ найдется представление (2). Положим $c_{\lambda, 0}:=c_{\lambda}$. Докажем, что существует $n, 0 \leqslant n \leqslant k$, для которого имеют место неравенства

$$
\max _{\lambda \in \Lambda}\left|c_{\lambda, n}\right| \leqslant c_{1}(p) c_{2}\left(\mu_{1}(\mathscr{D})\right) k^{-1 / p}|f|_{p}, \quad \sum_{\lambda \in \Lambda}\left|c_{\lambda, n}\right|^{p} \leqslant \sum_{\lambda \in \Lambda}\left|c_{\lambda, 0}\right|^{p}=|f|_{p}^{p} .
$$

Для каждого $m=1, \ldots, k$ для $n=m-1$ имеет место представление (3) и выполнено следующее условие: или $\max _{\lambda \in \Lambda}\left|c_{\lambda, m-1}\right| \leqslant k^{-1 / p}|f|_{p}$, и тогда в качестве $n$ можно взять $n=m-1$, или $\max _{\lambda \in \Lambda}\left|c_{\lambda, m-1}\right|>k^{-1 / p}|f|_{p}$, тогда согласно (5) будет выполняться условие на $\epsilon$ в лемме 3 . По лемме 3 будет иметь место представление (3) для $n=m$, и при этом с учетом (3) и (4)

$$
\begin{aligned}
0 & \leqslant \sum_{\lambda \in \Lambda}\left|c_{\lambda, m}\right|^{p} \leqslant \sum_{\lambda \in \Lambda}\left|c_{\lambda, m-1}\right|^{p}-2^{-p}\left(1-3 \mu_{1}(\mathscr{D})\right)^{p} \max _{\lambda \in \Lambda}\left|c_{\lambda, m-1}\right|^{p}=\cdots \\
& =\sum_{\lambda \in \Lambda}\left|c_{\lambda, 0}\right|^{p}-\sum_{l=1}^{m} 2^{-p}\left(1-3 \mu_{1}(\mathscr{D})\right)^{p} \max _{\lambda \in \Lambda}\left|c_{\lambda, l-1}\right|^{p} \\
& \leqslant|f|_{p}^{p}-m 2^{-p}\left(1-3 \mu_{1}(\mathscr{D})\right)^{p} \max _{\lambda \in \Lambda}\left|c_{\lambda, m}\right|^{p},
\end{aligned}
$$


что гарантирует выполнение (6) для $n=k$. Используя (6), оцениваем

$$
\begin{aligned}
\sum_{\lambda \in \Lambda}\left|c_{\lambda, n}\right|^{2} & =\sum_{\lambda \in \Lambda}\left|c_{\lambda, n}\right|^{p}\left|c_{\lambda, n}\right|^{2-p}=\left(\max _{\lambda \in \Lambda}\left|c_{\lambda, n}\right|\right)^{2-p} \sum_{\lambda \in \Lambda}\left|c_{\lambda, n}\right|^{p} \\
& \leqslant\left(c_{3}(p) c_{4}\left(\mu_{1}(\mathscr{D})\right) k^{-(2-p) / p}|f|_{p}^{2-p}\right)|f|_{p}^{p}=c_{3}(p) c_{4}\left(\mu_{1}(\mathscr{D})\right) k^{-2 / p+1}|f|_{p}^{2}
\end{aligned}
$$

Применяя неравенство треугольника, лемму 1 и (3), запишем

$$
\begin{aligned}
\left\|f_{k}\right\| \leqslant\left\|f_{n}\right\| \leqslant\left\|\sum_{\lambda \in \Lambda} c_{\lambda, n} g^{\lambda}\right\|+\left\|f_{\epsilon}\right\| & \leqslant\left(\left(1+2 \mu_{1}(\mathscr{D})\right) \sum_{\lambda \in \Lambda} c_{\lambda, n}^{2}\right)^{1 / 2}+\epsilon \\
& \leqslant C\left(p, \mu_{1}(\mathscr{D})\right) k^{-1 / p+1 / 2}|f|_{p}+\epsilon .
\end{aligned}
$$

Последнее неравенство, с учетом того, что $\epsilon$ может быть выбран сколь угодно малым, завершает доказательство теоремы.

ДоказАтельСтво теоремы 3. Из леммы 3 вытекает, что $\left|f_{m}\right|_{1} \leqslant|f|_{1}, m \geqslant 0$. Согласно лемме 3.5 из работы [2], для $m \geqslant 0$ имеет место неравенство

$$
\left|\left\langle f_{m}, g_{m+1}\right\rangle\right|=\sup _{g \in \mathscr{D}}\left|\left\langle f_{m}, g\right\rangle\right| \geqslant \frac{\left\|f_{m}\right\|^{2}}{\left|f_{m}\right|_{1}} \geqslant \frac{\left\|f_{m}\right\|^{2}}{|f|_{1}} .
$$

По определению ЧЖА

$$
\left\|f_{m+1}\right\|^{2}=\left\|f_{m}\right\|^{2}-\left\langle f_{m}, g_{m+1}\right\rangle^{2} \leqslant\left\|f_{m}\right\|^{2}-\left(\frac{\left\|f_{m}\right\|^{2}}{|f|_{1}}\right)^{2}=\left\|f_{m}\right\|^{2}\left(1-\frac{\left\|f_{m}\right\|^{2}}{|f|_{1}^{2}}\right) .
$$

Применяя лемму 3.4 из работы [2] для $a_{m}=\left\|f_{m-1}\right\|^{2}$ и $A=|f|_{1}^{2}$ с учетом неравенства $a_{1}=\left\|f_{0}\right\|^{2} \leqslant|f|_{1}^{2}$, получаем, что для $m \geqslant 1$

$$
\left\|f_{m-1}\right\|^{2}=a_{m} \leqslant A m^{-1}=|f|_{1}^{2} m^{-1} .
$$

\section{СПИСОК ЦИТИРОВАННОЙ ЛИТЕРАТУРЫ}

[1] V. N. Temlyakov, Acta Numer., 17 (2008), 235-409.[2] R. A. DeVore, V. N. Temlyakov, Adv. Comput. Math., 5:2-3 (1996), 173-187. [3] Е. Д. Лившиц, Изв. РАН. Сер. матем., 73:6 (2009), 125-144. [4] A. C. Gilbert, M. Muthukrishnan, M. J. Strauss, Proceedings of the Fourteenth Annual ACM-SIAM Symposium on Discrete Algorithms (Baltimore, MD, 2003), 2003, 243-252. [5] R. Gribonval, M. Nielsen, Independent Component Analysis and Blind Signal Separation, Lect. Notes Comput. Sci., 3195, Springer-Verlag, Berlin, 2004, 201-208. [6] J. A. Tropp, IEEE Trans. Inform. Theory, 50:10 (2004), 2231-2242. [7] D. L. Donoho, M. Elad, V. N. Temlyakov, IEEE Trans. Inform. Theory, 52:1 (2006), 6-18.

\section{Е. Д. Лившиц}

